\title{
Comparison of neuroendocrine dysfunction in patients with adamantinomatous and papillary craniopharyngiomas
}

\author{
YING FENG $^{1}$, MING NI $^{2}$, YONG-GANG WANG ${ }^{2}$ and LI-YONG ZHONG ${ }^{1}$ \\ Departments of ${ }^{1}$ Endocrinology and ${ }^{2}$ Neurosurgery, Beijing Tiantan Hospital, \\ Capital Medical University, Beijing 100050, P.R. China
}

Received March 13, 2018; Accepted June 15, 2018

DOI: $10.3892 /$ etm.2018.6953

\begin{abstract}
Craniopharyngiomas (CPs) are rare epithelial tumors mainly located in the sellar/parasellar region. They may be classified into two major histological subtypes, which exhibit clinical and pathological differences: Adamantinomatous $\mathrm{CP}$ (ACP) and papillary CP (PCP). The aim of the present study was to compare the neuroendocrine dysfunction between ACP and PCP prior to and after surgical resection. According to their pathological classification, the patients were assigned to the ACP group or the PCP group. The neuroendocrine dysfunction in these two CP subtypes was evaluated using a scoring method prior to and after surgery. A total of 741 patients with CPs were included in the present study, of which 622 were ACP and 119 were PCP. The scores on the tumor mass effect, hypothalamic dysfunction and pituitary-target gland axis dysfunction, as well as the incidence of central diabetes insipidus in the PCP group were all significantly higher than those in the ACP group, regardless of whether the surgery had been performed or not (all $\mathrm{P}<0.05)$. After surgery, the scores on the tumor mass effect were significantly decreased in the ACP and PCP patients (all $\mathrm{P}<0.05$ ), while the scores on hypothalamic dysfunction and pituitary-target gland axis dysfunction, as well as the incidence of central diabetes insipidus were all significantly increased in ACP and PCP patients (all $\mathrm{P}<0.05$ ). Prior to and after surgery, the PCP variant exhibited a greater damage to the neuroendocrine function compared with the ACP variant.
\end{abstract}

Correspondence to: Dr Li-Yong Zhong, Department of Endocrinology, Beijing Tiantan Hospital, Capital Medical University, 6 Tiantan West Street, Beijing 100050, P.R. China E-mail: zhongliyong@126.com

Abbreviations: CPs, craniopharyngiomas; ACP, adamantinomatous CP; PCP, papillary CP; WHO, World Health Organization; FSH, follicle-stimulating hormone; LH, luteinizing hormone; ACTH, adrenocorticotropic hormone; TSH, thyroid-stimulating hormone

Key words: adamantinomatous craniopharyngioma, papillary craniopharyngioma, neuroendocrine dysfunction, scoring methods

\section{Introduction}

Craniopharyngiomas (CPs) are rare, histologically benign class of epithelial tumors arising from embryological remnants of squamous epithelium of the craniopharyngeal duct, which are commonly located in the sellar and suprasellar regions $(1,2)$. They account for 1.2-4.0\% of all intracranial tumors (3). CPs are classified as grade I tumors by the World Health Organization (WHO) (4), and though considered histologically benign, they often present with a locally aggressive growth pattern. Furthermore, exceptional cases of malignant transformation have been reported (5). CPs pose numerous clinical challenges, most of which are neurological signs and symptoms, due to their intimate involvement and mass effect on adjacent structures, including the hypothalamus, pituitary gland and optic chiasm. The initial clinical manifestation at the time of diagnosis is often dominated by non-specific symptoms of elevated intracranial pressure, including headache and nausea. Further primary manifestations include visual impairment (i.e. visual acuity disorder and visual field defect; 62-84\%) and endocrine deficiencies (52-87\%) (6-8). At present, surgical resection followed by radiotherapy is the major treatment modality for CPs, although resection may contribute to a high risk of endocrine, neurological and hypothalamic post-operative complications due to the large lesion size, the location and its association with vital neurological structures $(2,8)$.

The estimated annual incidence of CPs is $0.5-2$ per 100,000 individuals, and $30-50 \%$ of all cases occur during childhood and adolescence (9). CPs may be classified into two major histological subtypes according to the WHO classification of tumors of the central nervous system (4): Adamantinomatous CP (ACP) and papillary CP (PCP). However, transitional or mixed forms have also been described (10). These two subtypes are pathologically distinct and ACPs are more common than PCPs (ratio, 9:1) (11). ACPs occur in patients of all ages, with a bimodal age distribution; the first peak occurs in the age window of 5-14 years and the second peak at 50-74 years (12). Histopathological features of ACPs are the formation of wet keratin nodules, a palisading basal layer of cells, loose aggregates of stellate cells, as well as the presence of large areas of regressive changes, i.e., multinucleated foreign body giant cells, hemosiderin deposits, cholesterol crystals, inflammation and calcification. Wet keratin is a hallmark of this variant (13). ACPs contain mutations in exon 3 of the gene encoding 
$\beta$-catenin (CTNNB1) in $92 \%$ of cases, leading to the overactivation of the WNT/ $\beta$-catenin signaling pathway, which has a crucial role in the tumorigenesis of ACP. However, the mutation of the $\beta$-catenin gene is not detectable in PCP (14).

By contrast, the papillary variant most frequent in adults aged 40-55 years (12). Histologically, PCPs are predominantly solid and are characterized by well-differentiated squamous epithelium with compact, monomorphic sheets covering fibrovascular cores with miniature capillaries and scattered immune cells, including macrophages and neutrophils. Areas with marked regressive changes, including cholesterol clefts, wet keratin, calcification and inflammation are absent. Ciliated epithelium and goblet cells are only occasionally observed, and the histological morphology resembles that of Rathke's cleft cysts with squamous metaplasia $(12,14,15)$. Mutations in B-Raf proto-oncogene, serine/threonine kinase (BRAF)V600E have been described in $95 \%$ of ACPs, causing constitutive activation of the mitogen-activated protein kinase pathway, thereby affecting cell division and differentiation (16).

CTNNB1 and BRAF mutations are mutually exclusive and specific for each subtype, indicating that ACP and PCP are two biologically distinct entities. They display differences in clinical manifestation, imaging characterization, histopathological morphology and recurrence rate $(12,17)$. In the present study, 741 cases of $\mathrm{CP}$ were assessed and a retrospective analysis of neuroendocrine dysfunction in ACP and PCP patients prior to and after surgical removal of the mass was performed.

\section{Materials and methods}

Patients. Patients with CP who underwent surgical resection at the Department of Neurosurgery of Beijing Tiantan Hospital affiliated to Capital Medical University (Beijing, China) between January 2011 and December 2016 were included in the present study. Subjects with an unknown pathological classification and those with a history of endocrine disorders, including primary hypothyroidism, adrenal insufficiency and hypogonadism, were excluded. The present retrospective study was performed in accordance with the declaration of Helsinki for research on humans and the protocol was approved by the Ethics Committee of Beijing Tiantan Hospital affiliated to Capital Medical University (Beijing, China).

Scoring methods. According to their pathological classification (4), the patients were divided into the ACP group and PCP group. A self-designed scoring method was used to evaluate the neuroendocrine dysfunction in these two CP subtypes prior to and after surgery.

The tumor mass effect was assessed by the three major symptoms, namely intracranial pressure (headache and nausea), loss of visual acuity and visual field defects. Each symptom was assigned a score of 0 (negative) or 1 (positive). The total score for the tumor mass effect therefore ranged from 0 to 3 .

Hypothalamic dysfunction was assessed based on the six manifestations, namely obesity and eating disorders, polydipsia and polyuria, sleep disorder, cognitive dysfunction and personality changes, imbalances in the regulation of body temperature and memory loss. Each manifestation was scored as 1 (positive) or 0 (negative). The total score for hypothalamic dysfunction ranged from 0 to 6 .
The pituitary hormones, including thyroid-stimulating hormone (TSH), adrenocorticotropic hormone (ACTH), luteinizing hormone ( $\mathrm{LH})$ /follicle-stimulating hormone (FSH), prolactin (PRL) and growth hormone, as well as the corresponding target gland (thyroid, adrenal and gonadal) hormones, including total triiodothyronine, free triiodothyronine, total thyroxine, free thyroxine, cortisol, testosterone, progesterone and estradiol, were measured to evaluate the function of the pituitary-target gland axis. Pituitary-target gland axis dysfunction was scored as follows: Low levels of TSH, or ACTH and cortisol, or FSH and LH were scored as 1, while normal levels scored as 0 to evaluate pituitary-thyroid, pituitary-adrenal or pituitary-gonadal axis dysfunction, respectively. The total score for pituitary-target gland axis dysfunction ranged from 0 to 3 .

In addition, the incidence of central diabetes insipidus was compared prior to and after the surgery within each group and between the two groups by measuring the 24-h urine volume and electrolytes.

Statistical analysis. All statistical analyses were performed by using SPSS version 20.0 software (IBM Corp., Armonk, NY, USA). The collected data were presented as the median (interquartile range) or $\mathrm{n}(\%)$. Outcomes were compared with the Wilcoxon rank-sum test for continuous variables and the chi-square test for categorical variables. $\mathrm{P}<0.05$ was considered to indicate a statistically significant difference.

\section{Results}

Age and sex distribution of ACP and PCP patients. A total of 741 patients with CPs were included in the present study, of which 622 had ACP and 119 PCP. In the ACP group, 333 patients $(53.5 \%)$ were males and $289(46.5 \%)$ were females. The median age of the patients was 14 years (interquartile range, 7-40 years). In the PCP group, 83 patients (69.7\%) were males and the remaining $36(30.3 \%)$ were females. The median age of the patients was 43 years (interquartile range, 29-51 years).

As presented in Fig. 1, a bimodal age distribution was observed in ACP patients, with peaks at 1-15 years and 40-45 years. Most of the ACP cases were of pediatric age. The PCP subtype primarily occurred in adults aged 40-53 years. The difference in age between the ACP and PCP groups was statistically significant $(\mathrm{P}<0.0001)$.

As presented in Table I, the male/female ratio in the ACP group was 1.15 and that in the PCP group was 2.31. Most of the CP patients were males. A significant difference in the sex ratio between the two $\mathrm{CP}$ subtypes was observed $(\mathrm{P}=0.001)$. In the ACP group, $52.7 \%$ of cases were aged $1-15$ years, while this age group only accounted for $11.8 \%$ of PCP cases; significant differences in the percentages of the age groups (1-15 and $>15$ years) were observed within and between the ACP and PCP groups $(\mathrm{P}<0.0001)$.

Comparison of neuroendocrine dysfunction of ACP and PCP patients. Prior to surgery, the patients with a score of 1-3 for the tumor mass effect accounted for $88.1 \%$ in the ACP group and $94.1 \%$ in the PCP group. The average score in the PCP group was significantly higher than that in the ACP group $(\mathrm{P}<0.0001)$. After surgery, the patients with a score of 1-3 for the tumor mass effect accounted for $53.5 \%$ in the ACP 
Table I. Age and sex distribution of ACP and PCP patients.

\begin{tabular}{lccc}
\hline Parameter & ACP $(\mathrm{n}=622)$ & PCP $(\mathrm{n}=119)$ & P-value \\
\hline Age (years) & $14(7-40)$ & $43(29-51)$ & $<0.0001$ \\
$1-15$ & $328(52.7)$ & $14(11.8)$ & $<0.0001$ \\
$>15$ & $294(47.3)$ & $105(88.2)$ & \\
Sex & & & \\
M & $333(53.5)$ & $83(69.7)$ & 0.001 \\
F & $289(46.5)$ & $36(30.3)$ & \\
\hline
\end{tabular}

Values are expressed as the median (interquartile range) or $\mathrm{n}(\%)$. $\mathrm{ACP}$, adamantinomatous craniopharyngioma; PCP, papillary craniopharyngioma; M, male; F, female.

group and $70.6 \%$ in the PCP group. The scores in the PCP group were significantly higher than those in the ACP group $(\mathrm{P}<0.0001$; Table II). After the surgery, the scores on the tumor mass effect in the ACP group $(\mathrm{P}<0.0001)$ and the PCP group $(\mathrm{P}<0.0001)$ were significantly decreased (Table III).

The patients with a score of 1-6 on hypothalamic dysfunction accounted for $34.4 \%$ in the ACP group and $44.5 \%$ in the PCP group prior to surgery. Patients with PCP had significantly higher scores than patients with ACP $(\mathrm{P}=0.040)$. After the surgery, the patients with a score of 1-6 on hypothalamic dysfunction accounted for $43.1 \%$ in the ACP group and $63.9 \%$ in the PCP group. The hypothalamic dysfunction scores in patients with PCP were significantly higher than those in patients with ACP $(\mathrm{P}<0.0001$; Table II). After the surgery, the scores on hypothalamic dysfunction in ACP $(\mathrm{P}=0.004)$ and $\mathrm{PCP}$ patients $(\mathrm{P}=0.008)$ were significantly increased (Table III).

The patients with a pituitary-target gland axis dysfunction score of 1-3 accounted for 55.8\% in the ACP group and $73.1 \%$ in the PCP group prior to surgery. The PCP patients had significantly higher scores than the ACP patients $(\mathrm{P}<0.0001)$. After the surgery, patients with a pituitary-target gland axis dysfunction score of 1-3 accounted for $91.3 \%$ in the ACP group and $98.3 \%$ in the PCP group. The scores in the PCP group were significantly higher than those in the ACP group $(\mathrm{P}<0.0001$; Table II). After the surgery, the scores on the pituitary-target gland axis dysfunction were significantly increased in the ACP $(\mathrm{P}<0.0001)$ and PCP groups $(\mathrm{P}<0.0001$; Table III $)$.

Central diabetes insipidus occurred in $24.6 \%$ of cases with $\mathrm{ACP}$ and $34.5 \%$ of cases with PCP prior to surgery. After the surgery, $42.9 \%$ of ACP patients and $65.5 \%$ of PCP patients presented with central diabetes insipidus. Prior to and after the surgery, the prevalence of central diabetes insipidus in the PCP group was significantly higher than that in the ACP group $(\mathrm{P}=0.030$ and $\mathrm{P}<0.0001$, respectively). After surgical removal of the mass, the incidence of central diabetes insipidus increased significantly from 24.6 to $42.9 \%$ in the ACP group $(\mathrm{P}<0.0001)$, and from 34.5 to $65.5 \%$ in the PCP group $(\mathrm{P}<0.0001)$.

\section{Discussion}

CPs are non-neuroepithelial intracerebral neoplasms with a variable histological appearance. They frequently behave aggressively with invasion of the surrounding vital neurologic structures, including the optic nerve and hypothalamic-pituitary axes, consequently causing hypopituitarism and hypothalamic syndromes (7). Depending on patient age, location and size of the tumor, as well as extension to adjacent structures, the clinical presentation of CPs is variable. Accordingly, the management of CPs remains a challenge for neurosurgeons. At present, CPs may be treated and controlled but not cured, and studies have reported that recurrence rates after radical gross total resection of $\mathrm{CP}$ with hypothalamic involvement were similar to the progression rates after limited surgery, resulting in residual tumor (18). In addition, due to the inflammatory adhesions between the tumor and surrounding brain tissue, further damage may occur during surgery and/or radiotherapy. The surgical removal of the tumor tissue may cause irreversible damage to the hypothalamic-pituitary target gland axes, affecting the neuroendocrine function. Most patients require lifelong pituitary hormone replacement therapy. Visual impairment, hormonal disturbances of hypothalamus and pituitary glands, obesity, cognitive impairment and personality changes are common complications caused not only by the growth of the tumor but also frequently as a consequence of treatment with surgery and/or radiation therapy (15). Pre-operative demonstration of the extensive adherence of the large tumor to hypothalamic structures may be associated with a poor endocrinological outcome after surgical resection (19).

ACP and PCP subtypes are completely distinctive entities, which differ in their histological, genetic and clinical behavior. Esheba and Hassan (20) have demonstrated that $\beta$-catenin mutations and/or nuclear accumulation are diagnostic hallmarks of ACP and are helpful in the differential diagnosis between these two subtypes. The histopathological classification has clinical significance and prognostic value. Szeifert et al (17) have indicated that the pathological classification of CPs may influence the post-operative outcome, and the ACP subtype is associated with a high recurrence rate and poor prognosis. The aim of the present study was to compare the neuroendocrine dysfunction prior to and after surgical resection within and between the two histopathological types of CP.

The present study included 622 cases of ACP and 119 cases of PCP. CPs may be detected at any age. The age distribution of ACPs exhibited a bimodal distribution with peak incidence rates in children and adolescents aged 1-15 years and adults aged 40-45 years. Apart from a small proportion of pediatric cases, PCPs almost exclusively occur in adults aged 40-53 years. The difference in age between the ACP and PCP groups was statistically significant $(\mathrm{P}<0.0001)$. The above results were consistent with those of a previously published study (12). ACPs are the most common form of non-neuroepithelial neoplasm in children, accounting for $5-10 \%$ of intracranial tumors in this population (21). An equal sex ratio has been reported in population-based studies from the United States (22) and Finland (23). However, in the present study, males were more commonly affected than females $(\mathrm{P}=0.001)$. An admission bias may have been present, as the current study was a single-center retrospective cohort study.

Neuroendocrinological evaluation was performed during the pre- and post-operative periods using a self-designed scoring method. The results of the present study indicated that the scores on the tumor mass effect, hypothalamic dysfunction and pituitary-target gland axis dysfunction, as well as 
Table II. Comparison of neuroendocrine dysfunction prior to and after the surgical removal of mass between the two groups.

\begin{tabular}{|c|c|c|c|c|c|c|}
\hline \multirow[b]{2}{*}{ Parameter/score } & \multicolumn{3}{|c|}{ Prior to surgery } & \multicolumn{3}{|c|}{ After surgery } \\
\hline & $\mathrm{ACP}$ & PCP & P-value & $\mathrm{ACP}$ & PCP & P-value \\
\hline Tumor mass effect & $1.74 \pm 0.99$ & $2.11 \pm 0.93$ & $<0.0001$ & $1.05 \pm 1.02$ & $1.37 \pm 0.97$ & $<0.0001$ \\
\hline 0 & 74 (11.9) & 7 (5.9) & & $289(46.5)$ & 35 (29.4) & \\
\hline 1 & $182(29.3)$ & $24(20.1)$ & & $37(5.9)$ & $12(10.1)$ & \\
\hline 2 & $195(31.4)$ & $37(31.1)$ & & $275(44.2)$ & $65(54.6)$ & \\
\hline 3 & $171(27.4)$ & $51(42.9)$ & & $21(3.4)$ & $7(5.9)$ & \\
\hline Hypothalamic dysfunction & $0.47 \pm 0.79$ & $0.62 \pm 0.87$ & 0.040 & $0.60 \pm 0.92$ & $0.88 \pm 0.97$ & $<0.0001$ \\
\hline 0 & $408(65.6)$ & $66(55.5)$ & & $354(56.9)$ & $43(36.1)$ & \\
\hline 1 & $158(25.4)$ & $40(33.6)$ & & $213(34.2)$ & $61(51.3)$ & \\
\hline 2 & $41(6.6)$ & $7(5.9)$ & & $23(3.7)$ & $7(5.9)$ & \\
\hline 3 & $9(1.4)$ & $4(3.4)$ & & $18(2.9)$ & $3(2.5)$ & \\
\hline $4-6$ & $6(1.0)$ & $2(1.6)$ & & $14(2.3)$ & $5(4.2)$ & \\
\hline Pituitary-target gland dysfunction & $0.94 \pm 1.00$ & $1.63 \pm 1.22$ & $<0.0001$ & $1.94 \pm 0.95$ & $2.54 \pm 0.77$ & $<0.0001$ \\
\hline 0 & $275(44.2)$ & $32(26.9)$ & & $54(8.7)$ & $2(1.7)$ & \\
\hline 1 & $170(27.3)$ & $22(18.5)$ & & $135(21.7)$ & $14(11.8)$ & \\
\hline 2 & $118(19.0)$ & $23(19.3)$ & & $225(36.2)$ & $21(17.6)$ & \\
\hline 3 & $59(9.5)$ & $42(35.3)$ & & $208(33.4)$ & $82(68.9)$ & \\
\hline Central diabetes insipidus & $153(24.6)$ & $41(34.5)$ & 0.030 & $267(42.9)$ & $78(65.5)$ & $<0.0001$ \\
\hline
\end{tabular}

Values are expressed as n (\%). ACP, adamantinomatous craniopharyngioma; PCP, papillary craniopharyngioma.

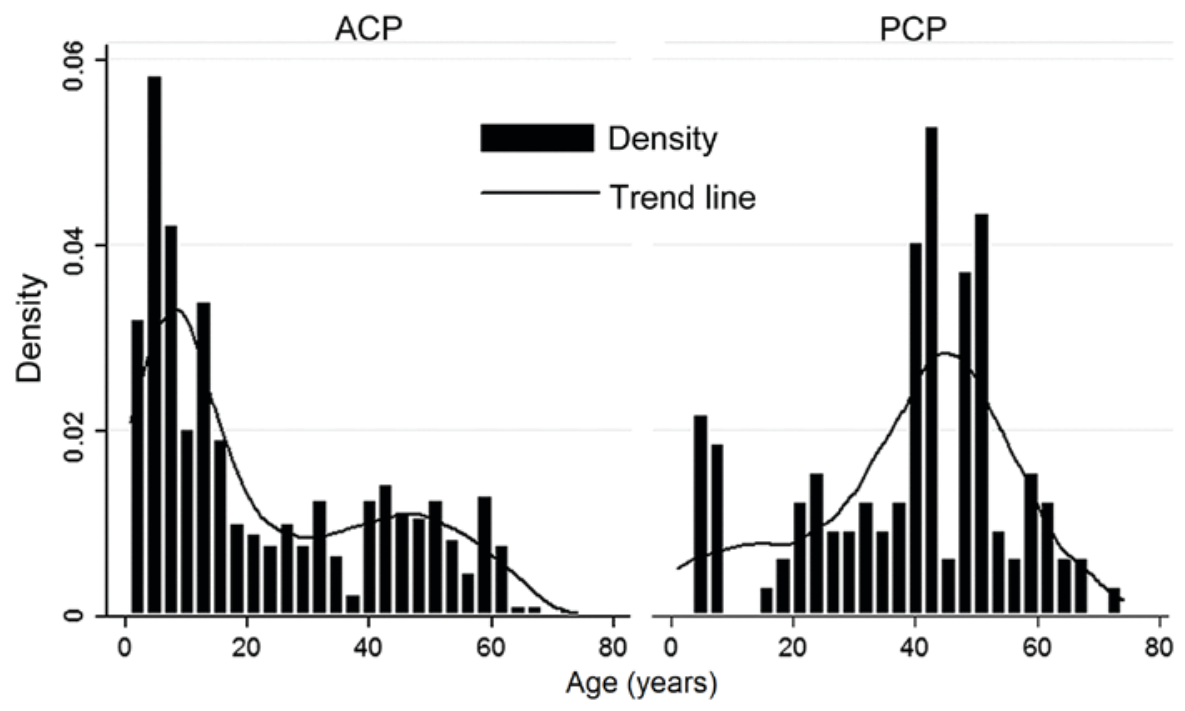

Figure 1. Age distribution of ACP and PCP patients. ACP, adamantinomatous craniopharyngioma; PCP, papillary craniopharyngioma.

the incidence of central diabetes insipidus in PCP were all significantly higher than those in ACP regardless of whether surgery had been performed (all $\mathrm{P}<0.05)$. This indicated that the symptoms associated with neuroendocrine dysfunction were more severe among PCP patients with a greater degree of damage. The tumor mass effect scores in ACP and PCP patients were decreased after surgery (all $\mathrm{P}<0.05$ ). Clinical symptoms arising from the tumor mass effect were relieved. Visual impairment as an initial clinical symptom of CPs is reported in more than half of affected patients, and vision was reported to have improved post-operatively in $41-48 \%$ of patients with pre-operative visual impairment (24).

However, in the present study, the scores on the hypothalamic dysfunction and the pituitary-target gland axis dysfunction, as well as the incidence of central diabetes insipidus in ACP and PCP patients were all increased after surgical removal of the mass (all $\mathrm{P}<0.05$ ). The clinical symptoms of hypothalamic dysfunction and pituitary-target gland axis dysfunction were aggravated in these two subtypes. Most patients with CPs presented with multiple deficits of 
Table III. Comparison of neuroendocrine dysfunction prior to and after the surgical removal of mass within each group.

\begin{tabular}{|c|c|c|c|c|c|c|}
\hline \multirow[b]{2}{*}{ Parameter } & \multicolumn{3}{|c|}{ ACP group } & \multicolumn{3}{|c|}{ PCP group } \\
\hline & $\begin{array}{l}\text { Prior to } \\
\text { surgery }\end{array}$ & After surgery & P-value & $\begin{array}{l}\text { Prior to } \\
\text { surgery }\end{array}$ & After surgery & P-value \\
\hline Tumor mass effect & $1.74 \pm 0.99$ & $1.05 \pm 1.02$ & $<0.0001$ & $2.11 \pm 0.93$ & $1.37 \pm 0.97$ & $<0.0001$ \\
\hline 0 & $74(11.9)$ & $289(46.5)$ & & $7(5.9)$ & $35(29.4)$ & \\
\hline 1 & $182(29.3)$ & $37(5.9)$ & & $24(20.1)$ & $12(10.1)$ & \\
\hline 2 & $195(31.4)$ & $275(44.2)$ & & $37(31.1)$ & $65(54.6)$ & \\
\hline 3 & $171(27.4)$ & $21(3.4)$ & & $51(42.9)$ & $7(5.9)$ & \\
\hline Hypothalamic dysfunction & $0.47 \pm 0.79$ & $1.60 \pm 0.92$ & 0.004 & $0.62 \pm 0.87$ & $0.88 \pm 0.98$ & 0.008 \\
\hline 0 & $408(65.6)$ & $354(56.9)$ & & $66(55.5)$ & $43(36.1)$ & \\
\hline 1 & $158(25.4)$ & $213(34.2)$ & & $40(33.6)$ & $61(51.3)$ & \\
\hline 2 & $41(6.6)$ & $23(3.7)$ & & $7(5.9)$ & $7(5.9)$ & \\
\hline 3 & $9(1.4)$ & $18(2.9)$ & & $4(3.4)$ & $3(2.5)$ & \\
\hline $4-6$ & $6(1.0)$ & $14(2.3)$ & & $2(1.6)$ & $5(4.2)$ & \\
\hline $\begin{array}{l}\text { Pituitary-target gland } \\
\text { dysfunction }\end{array}$ & $0.94 \pm 1.00$ & $1.94 \pm 0.95$ & $<0.0001$ & $1.63 \pm 1.22$ & $2.54 \pm 0.77$ & $<0.0001$ \\
\hline 0 & $275(44.2)$ & $54(8.7)$ & & $32(26.9)$ & $2(1.7)$ & \\
\hline 1 & $170(27.3)$ & $135(21.7)$ & & $22(18.5)$ & $14(11.8)$ & \\
\hline 2 & $118(19.0)$ & $225(36.2)$ & & $23(19.3)$ & $21(17.6)$ & \\
\hline 3 & $59(9.5)$ & $208(33.4)$ & & $42(35.3)$ & $82(68.9)$ & \\
\hline Central diabetes insipidus & $153(24.6)$ & 267 (42.9) & $<0.0001$ & $41(34.5)$ & $78(65.5)$ & $<0.0001$ \\
\hline
\end{tabular}

Values are expressed as n (\%). ACP, adamantinomatous craniopharyngioma; PCP, papillary craniopharyngioma.

hypothalamic-pituitary function at the time of diagnosis. Surgical removal of the mass may further affect hypothalamic and pituitary function due to the proximity of the tumor to the hypothalamic-pituitary axes, aggravating hypothalamic dysfunction and endocrine disorders; in severe cases, the condition may be life-threatening $(24,25)$. Hypothalamic neuroendocrine dysfunction, including obesity, behavioral changes, disturbed circadian rhythm and sleep disorder, imbalances in the regulation of the body temperature, polydipsia and memory loss, was reported in $35 \%$ of CP patients at diagnosis (1). The rate of hypothalamic dysfunction markedly increases following radical surgery to up to $65-80 \%$ (26).

Pituitary hormone deficiencies are common in patients with CPs. Prior to surgery, $40-87 \%$ of patients have been reported to have at least one hormonal deficit, and post-surgical pituitary hormone deficiencies occur in up to $85-95 \%$ of patients. Central diabetes insipidus frequently occurs prior to $(17-27 \%$ of cases) or after surgery (40-93\% of cases) (6). Anti-diuretic hormone is synthesized in the supraoptic nucleus and the paraventricular nucleus of the hypothalamus, and is transported through the pituitary stalk and stored in the posterior pituitary gland. This hormone is released into the circulation in response to changes in plasma osmotic pressure, which regulates the amount of urine. Patients with ACP or PCP may have central diabetes insipidus due to the tumor compressing the pituitary stalk. The present study indicated that surgery may damage or even sever the pituitary stalk, which may aggravate central diabetes insipidus. This is also supported by the post-operative images displaying an abnormal structure or absence of the pituitary stalk (results not shown).

The present study compared the neuroendocrine dysfunction prior to and after surgical treatment between and within the ACP and PCP groups. Systematic evaluation of the functional status of hypothalamic-pituitary-target gland axes in different histological types of CPs after removal of the tumor in situ may contribute to the formulation of individualized target hormone replacement therapy protocols and neuroendocrine rehabilitation therapy. At the genetic level, the apparent mutual exclusivity of CTNNB1 and BRAFV600E mutations in the CP variants is a potential target for pharmaceutical therapy (13).

Of note, the present study has certain limitations. The present study included more male than female cases, while previous studies reported equal sex ratios. This may be caused by the high male sex ratio at birth during the past decades in China. Another possible reason is that due to the female discrimination in rural areas of China, females with severe diseases are less likely to be treated locally and referred to higher-level hospitals. In addition, due to the retrospective nature of the present study, bias may have been introduced in the results.

In conclusion, the PCP variant exhibited a greater damage to the neuroendocrine function compared with the ACP variant prior to and after surgery. Further study of neuroendocrine dysfunction in the two histologic subtypes of CPs with larger sample sizes is warranted to confirm and expand the results of the present study. Besides the removal of the mass effect, the treatment of patients with $\mathrm{CP}$ requires to focus on and monitor 
long-term changes in neuroendocrine function. To improve the quality of life of affected patients, functional reconstruction of the hypothalamic-pituitary-gonadal axis should be emphasized.

\section{Acknowledgements}

Not applicable.

\section{Funding}

The present study was supported by the Characteristic Project on Capital Clinical Research, Beijing Municipal Science and Technology Committee (no: Z181100001718122).

\section{Availability of data and materials}

The analysed data sets generated during the present study are available from the corresponding author on reasonable request.

\section{Authors' contributions}

YF collected and analyzed clinical data, and prepared the manuscript; LZ designed the trial and prepared the manuscript; and $\mathrm{MN}$ and YW collected clinical data. The final version of the manuscript has been read and approved by all authors, and each author believes that the manuscript represents honest work.

\section{Ethical approval and consent to participate}

The present study was approved by the Ethics Committee of Beijing Tiantan Hospital affiliated to Capital Medical University (Beijing, China).

\section{Patient consent for publication}

Not applicable.

\section{Competing interests}

The authors declare that they have no competing interests.

\section{References}

1. Elliott RE and Wisoff JH: Surgical management of giant pediatric craniopharyngiomas. J Neurosurg Pediatr 6: 403-416, 2010.

2. Poretti A, Grotzer MA, Ribi K, Schonle E and Boltshauser E: Outcome of craniopharyngioma in children: Long-term complications and quality of life. Dev Med Child Neurol 46: 220-229, 2004

3. Qi ST, Zhou J, Pan J, Zhang C, Silky C and Yan XR: Epithelial-mesenchymal transition and clinicopathological correlation in craniopharyngioma. Histopathology 61: 711-725, 2012.

4. Louis DN, Ohgaki H, Wiestler OD, Cavenee WK, Burger PC, Jouvet A, Scheithauer BW and Kleihues P: The 2007 WHO classification of tumours of the central nervous system. Acta Neuropathol 114: 97-109, 2007.

5. Aquilina K, Merchant TE, Rodriguez-Galindo C, Ellison DW Sanford RA and Boop FA: Malignant transformation of irradiated craniopharyngioma in children: Report of 2 cases. J Neurosurg Pediatr 5: 155-161, 2010.

6. Daubenbuchel AM and Muller HL: Neuroendocrine disorders in pediatric craniopharyngioma patients. J Clin Med 4: 389-413, 2015.
7. Balcazar-Hernandez L, Vargas-Ortega G, Valverde-Garcia Y, Mendoza-Zubieta V and Gonzalez-Virla B: Anorexia-cachexia syndrome-like hypothalamic neuroendocrine dysfunction in a patient with a papillary craniopharyngioma. Endocrinol Diabetes Metab Case Rep 2017, 2017.

8. Caldarelli M, Massimi L, Tamburrini G, Cappa M and Di Rocco C: Long-term results of the surgical treatment of craniopharyngioma: The experience at the Policlinico Gemelli, Catholic University, Rome. Childs Nerv Syst 21: 747-757, 2005.

9. Nielsen EH, Feldt-Rasmussen U, Poulsgaard L, Kristensen LO, Astrup J, Jørgensen JO, Bjerre P, Andersen M, Andersen C, Jørgensen J, et al: Incidence of craniopharyngioma in Denmark $(\mathrm{n}=189)$ and estimated world incidence of craniopharyngioma in children and adults. J Neurooncol 104: 755-763, 2011.

10. Karavitaki N: Management of craniopharyngiomas. J Endocrinol Invest 37: 219-228, 2014.

11. Lubuulwa J and Lei T: Pathological and topographical classification of craniopharyngiomas: A literature review. J Neurol Surg Rep 77: e121-e127, 2016.

12. Holsken A, Sill M, Merkle J, Schweizer L, Buchfelder M, Flitsch J, Fahlbusch R, Metzler M, Kool M, Pfister SM, et al: Adamantinomatous and papillary craniopharyngiomas are characterized by distinct epigenomic as well as mutational and transcriptomic profiles. Acta Neuropathol Commun 4: 20 , 2016.

13. Martinez-Barbera JP: Molecular and cellular pathogenesis of adamantinomatous craniopharyngioma. Neuropathol Appl Neurobiol 41: 721-732, 2015.

14. Martinez-Barbera JP and Buslei R: Adamantinomatous craniopharyngioma: Pathology, molecular genetics and mouse models. J Pediatr Endocrinol Metab 28: 7-17, 2015.

15. Brastianos PK and Santagata S: ENDOCRINE TUMORS: BRAF V600E mutations in papillary craniopharyngioma. Eur J Endocrinol 174: R139-R144, 2016.

16. Brastianos PK, Taylor-Weiner A, Manley PE, Jones RT, Dias-Santagata D, Thorner AR, Lawrence MS, Rodriguez FJ, Bernardo LA, Schubert L, et al: Exome sequencing identifies BRAF mutations in papillary craniopharyngiomas. Nat Genet 46: 161-165, 2014

17. Szeifert GT, Sipos L, Horvath M, Sarker MH, Major O, Salomváry B, Czirják S, Bálint K, Slowik F, Kolonics L, et al: Pathological characteristics of surgically removed craniopharyngiomas: Analysis of 131 cases. Acta Neurochir (Wien) 124: 139-143, 1993.

18. Muller HL: Craniopharyngioma: Long-term consequences of a chronic disease. Expert Rev Neurother 15: 1241-1244, 2015.

19. Lopez-Serna R, Gomez-Amador JL, Barges-Coll J, Nathal-Vera E, Revuelta-Gutiérrez R, Alonso-Vanegas M, Ramos-Peek M and Portocarrero-Ortiz L: Treatment of craniopharyngioma in adults: Systematic analysis of a 25 -year experience. Arch Med Res 43: 347-355, 2012.

20. Esheba GE and Hassan AA: Comparative immunohistochemical expression of $\beta$-catenin, EGFR, ErbB2, and p63 in adamantinomatous and papillary craniopharyngiomas. J Egypt Natl Canc Inst 27: 139-145, 2015 .

21. Puget S, Garnett M, Wray A, Grill J, Habrand JL, Bodaert N, Zerah M, Bezerra M, Renier D, Pierre-Kahn A and Sainte-Rose C: Pediatric craniopharyngiomas: Classification and treatment according to the degree of hypothalamic involvement. J Neurosurg 106: 3-12, 2007.

22. Bunin GR, Surawicz TS, Witman PA, Prestonmartin S, Davis F and Bruner JM: The descriptive epidemiology of craniopharyngioma. J Neurosurg 89: 547-551, 1998.

23. Sorva R and Heiskanen O: Craniopharyngioma in Finland. A study of 123 cases. Acta Neurochir (Wien) 81: 85-89, 1986.

24. Müller HL: Consequences of craniopharyngioma surgery in children. J Clin Endocrinol Metab 96: 1981-1991, 2011.

25. Muller HL: Craniopharyngioma and hypothalamic injury: Latest insights into consequent eating disorders and obesity. Curr Opin Endocrinol Diabetes Obes 23: 81-89, 2016.

26. Scarfone RJ, Loiselle JM, Wiley JF II, Decker JM, Henretig FM and Joffe MD: Nebulized dexamethasone versus oral prednisone in the emergency treatment of asthmatic children. Ann Emerg Med 26: 480-486, 1995.

This work is licensed under a Creative Commons Attribution-NonCommercial-NoDerivatives 4.0 International (CC BY-NC-ND 4.0) License. 